\title{
Phase Equilibria of the Modified Buckingham Exponential-6 Potential from Hamiltonian Scaling Grand Canonical Monte Carlo
}

\author{
Jeffrey R. Errington and Athanassios Z. Panagiotopoulos* \\ School of Chemical Engineering \\ Cornell University \\ Ithaca, NY 14853 \\ and \\ Institute for Physical Science and Technology \\ and Department of Chemical Engineering, \\ University of Maryland \\ College Park, MD 20742
}

2 April, 1998

\footnotetext{
To whom correspondence should be addressed at the University of Maryland. E-mail: thanos@ipst.umd.edu
} 


\section{Abstract}

A novel method, Hamiltonian scaling grand canonical Monte Carlo, has been used to determine the phase behavior of twelve variations of the modified Buckingham exponential-6 potential. Hamiltonian scaling grand canonical Monte Carlo enables the determination of thermodynamic properties of several related potential models from a single set of simulations. The main advantage of the method is that by appropriate selection of the chemical potential and temperature for each Hamiltonian, the densities that are sampled are relevant for multiple Hamiltonians, thus increasing the simulation efficiency. The method was combined with mixed-field finite-size scaling to determine the critical parameters and phase coexistence curves of several modified Buckingham exponential-6 potentials to a high level of accuracy. In particular, the critical parameters of the models have been calculated to within $0.3 \%$ for the critical temperature and $1 \%$ for the critical density and pressure. A new potential for methane based on the Buckingham exponential- 6 model is presented. The vapor pressure curve of methane from the Buckingham exponential-6 potential model is much more accurate than is possible with the Lennard-Jones potential model. 


\section{Introduction}

Phase equilibria of real fluids and model systems are of significant interest to scientists and engineers. Not long ago the determination of phase equilibria for even the simplest of systems was an arduous task. In recent years, advances in simulation methodologies have made it possible to obtain accurate results for the phase behavior of a broad range of systems.

Early methods for determining phase equilibria involved the formulation of an equation of state from simulations at numerous state points ${ }^{1.4}$. The introduction of the Gibbs ensemble technique ${ }^{5.7}$ greatly simplified this procedure by enabling calculations of the properties of two coexisting phases at a given temperature from a single simulation. Although a significant improvement over previous techniques, the method is difficult to apply near critical points. More recently, histogram reweighting grand canonical Monte Carlo simulations ${ }^{8,9}$, combined with mixed-field finite-size scaling concepts ${ }^{10,11}$, have been used to determine critical parameters and phase behavior for model systems to unprecedented accuracy. During a grand canonical ensemble simulation a histogram is collected of the frequency of observing the system with a given particle number and energy. Properties of the system can then be evaluated for a range of state points by rescaling the histogram to a different chemical potential and temperature than originally simulated. 
These methodological advances have brought molecular simulation to the point where it can be used to predict reliably the thermophysical properties of complex, industrially important fluids. The limiting factor is the lack of accurate intermolecular potential models for real fluids. The determination of these potential models inevitably involves the fitting of model parameters to experimental data ${ }^{12.14}$. The fitting process requires a search through parameter space for the set of parameters that best describe a collection of properties over a wide range of state points. This search can quickly become impractical if many state points and parameters are examined.

A promising, but previously unexplored, approach for reducing the computational time required for optimizing intermolecular potential parameters is to take advantage of "thermodynamic scaling Monte Carlo" concepts ${ }^{15.19}$ proposed by Valleau. These methods make use of an unphysical, nonBoltzmann distribution to simulate multiple state points and/or Hamiltonians (potential models) in a single simulation. The ideas have recently been extended by Kiyohara et al. ${ }^{20}$ who have formulated temperature and Hamiltonian scaling Gibbs ensemble calculations. The main difficulty with thermodynamic scaling Monte Carlo is finding a sampling distribution that explores configurations relevant to multiple state points and Hamiltonians. This sampling distribution is usually obtained from exploratory simulations before the production phase. The number of exploratory simulations needed depends heavily on the relative difference between state points and Hamiltonians being studied. Despite the 
extra complexity, thermodynamic scaling Monte Carlo is still more efficient than conventional Monte Carlo in many cases.

In this work we propose a combination of thermodynamic and Hamiltonian scaling with grand canonical histogram reweighting Monte Carlo. The idea is to simulate multiple Hamiltonians in a single simulation, with each one assigned a temperature and activity such that all sample similar densities. By doing so, most configurations generated are relevant for several Hamiltonians and the sampling efficiency is increased. To demonstrate the method we determine the phase behavior for multiple variations of the modified Buckingham exponential-6 potential ${ }^{21}$.

The modified Buckingham exponential-6 potential is a four-parameter model of the form

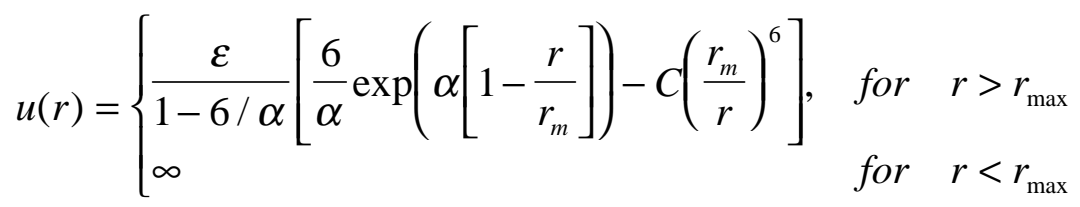

where $\varepsilon, r_{m}, \alpha$, and $C$ are model parameters. Parameter $r_{m}$ is the radial distance at which the potential is a minimum. The cutoff distance $r_{\max }$ is the smallest positive value for which $d u(r) / d r=0$ and is obtained by iterative solution of equation (1). The reason a cutoff distance is required is that at very short distances, the original Buckingham exponential-6 potential becomes negative. While canonical-ensemble Monte Carlo or molecular dynamics simulations never sample the unphysical attractive region, this is not the case on trial insertions in grand canonical simulations. The radial distance for which $u(r)=0$ can also be 
computed from equation (1). It is denoted by $\sigma$ and is used to normalize the thermodynamic properties, for consistency with the commonly used LennardJones potential. In a previous study, Dodd and Sandler ${ }^{22}$ formulated an equation of state for the Buckingham exponential-6 potential based on canonical ensemble calculations, with $C=1.0$ and $\alpha=14$, over a wide range of temperatures and densities. Their work was later extended to mixtures of Buckingham exponential-6 particles ${ }^{23}$. In the present paper we determine the phase behavior for a range of $\alpha$ and $C$ values.

The plan of the paper is as follows. In the next section we present the theoretical framework of Hamiltonian scaling grand canonical histogram reweighting Monte Carlo and describe its practical implementation. Then, we present our results of the critical parameters and phase behavior for a series of $\alpha$ and $C$ values. Finally, a new potential for methane based on the Buckingham exponential-6 model is presented and the results compared to experimental data and calculations based on the Lennard-Jones potential model.

\section{Simulation Methods}

\section{Hamiltonian Scaling Grand Canonical Monte Carlo}

In the grand canonical ensemble particles are created and annihilated in a simulation cell of volume $V$ under periodic boundary conditions. For each Hamiltonian $n$ a value of the chemical potential $\mu_{n}$ and temperature $T_{n}$ are set. If 
the coordinates of particles of the system are written as $\mathbf{r}^{N}$, the grand canonical ensemble sampling distribution $\Psi_{n}\left(\mathbf{r}^{N}\right)$ for a given Hamiltonian $n$ is

$$
\begin{aligned}
\Psi_{n}\left(\mathbf{r}^{N}\right) & =\frac{V^{N}}{\Lambda_{n}^{3 N} N !} \exp \left(\beta_{n} \mu_{n} N-\beta_{n} E_{n}\left(\mathbf{r}^{N}\right)\right) \\
& =\Omega\left(N, V, E_{n}\right) \exp \left(\beta_{n} \mu_{n} N-\beta_{n} E_{n}\left(\mathbf{r}^{N}\right)\right)
\end{aligned}
$$

where $\Lambda_{n}$ is the de Broglie wavelength $\left(\Lambda_{n}=\sqrt{h^{2} /\left(2 \pi m k_{B} T_{n}\right)}\right), \beta_{n}$ is the reciprocal temperature $\left(\beta_{n}=1 / k_{B} T_{n}\right.$, where $k_{B}$ is Boltzmann's constant), and $E_{n}$ is the total configurational energy. The thermodynamic average of any property, $X$, evaluated while sampling with the conventional distribution $\Psi_{n}\left(\mathbf{r}^{N}\right)$ is

$$
\left\langle X_{n}\right\rangle=\frac{\sum_{N=0}^{\infty} \int_{V} X_{n}\left(\mathbf{r}^{N}\right) \Psi_{n}\left(\mathbf{r}^{N}\right) d \mathbf{r}^{N}}{\sum_{N=0}^{\infty} \int_{V} \Psi_{n}\left(\mathbf{r}^{N}\right) d \mathbf{r}^{N}}=\left\langle X_{n}\left(\mathbf{r}^{N}\right)\right\rangle_{\Psi_{n}\left(\mathbf{r}^{N}\right)} .
$$

When a non-conventional sampling distribution $\pi\left(\mathbf{r}^{N}\right)$ is introduced the average is determined from

$$
\left\langle X_{n}\right\rangle=\frac{\sum_{N=0}^{\infty} \int_{V} \pi\left(\mathbf{r}^{N}\right) X_{n}\left(\mathbf{r}^{N}\right) \frac{\Psi_{n}\left(\mathbf{r}^{N}\right)}{\pi\left(\mathbf{r}^{N}\right)} d \mathbf{r}^{N}}{\sum_{N=0}^{\infty} \int_{V} \pi\left(\mathbf{r}^{N}\right) \frac{\Psi_{n}\left(\mathbf{r}^{N}\right)}{\pi\left(\mathbf{r}^{N}\right)} d \mathbf{r}^{N}}=\frac{\left\langle X_{n}\left(\mathbf{r}^{N}\right) \frac{\Psi_{n}\left(\mathbf{r}^{N}\right)}{\pi\left(\mathbf{r}^{N}\right)}\right\rangle_{\pi\left(\mathbf{r}^{N}\right)}}{\left\langle\frac{\Psi_{n}\left(\mathbf{r}^{N}\right)}{\pi\left(\mathbf{r}^{N}\right)}\right\rangle_{\pi\left(\mathbf{r}^{N}\right)}},
$$

where the \langle\rangle$_{\chi\left(\mathrm{r}^{N}\right)}$ brackets above indicate an average over all configurations sampled with the $\chi\left(\mathbf{r}^{N}\right)$ distribution. 
While equation (4) places no restrictions on the choice of $\pi\left(\mathbf{r}^{N}\right)$, its selection is critical. A $\pi\left(\mathbf{r}^{N}\right)$ must be chosen such that all regions of phase space relevant to each of the Hamiltonians are visited with adequate frequency. Unfortunately, there is no way of knowing an appropriate $\pi\left(\mathbf{r}^{N}\right)$ a priori. A suitable choice for $\pi\left(\mathbf{r}^{N}\right)$, proposed by Valleau ${ }^{19}$, is a weighted linear combination of the conventional sampling distributions of all the Hamiltonians.

$$
\pi\left(\mathbf{r}^{N}\right)=\sum_{n} W_{n} \Psi_{n}\left(\mathbf{r}^{N}\right)
$$

Where the $W_{n}$ are, yet unknown, sampling weights for each of the Hamiltonians. This choice has convenient properties that allow for the straightforward determination of the sampling weights. The relative degree to which each Hamiltonian is sampled throughout a simulation is proportional to $\sum_{N=0}^{\infty} \int_{V} W_{n} \Psi_{n}\left(\mathbf{r}^{N}\right) d \mathbf{r}^{N}$. This property is closely related to the grand partition function $\Xi$. The grand partition functions of two Hamiltonians are related as follows

$$
\frac{\Xi_{i}}{\Xi_{j}}=\frac{\sum_{N=0}^{\infty} \int_{V} \Psi_{i}\left(\mathbf{r}^{N}\right) d \mathbf{r}^{N}}{\sum_{N=0}^{\infty} \int_{V} \Psi_{j}\left(\mathbf{r}^{N}\right) d \mathbf{r}^{N}}=\frac{\left\langle\frac{\Psi_{i}\left(\mathbf{r}^{N}\right)}{\pi\left(\mathbf{r}^{N}\right)}\right\rangle_{\pi\left(\mathbf{r}^{N}\right)}}{\left\langle\frac{\Psi_{j}\left(\mathbf{r}^{N}\right)}{\pi\left(\mathbf{r}^{N}\right)}\right\rangle_{\pi\left(\mathbf{r}^{N}\right)}} .
$$

This suggests that the ratio of sampling weights should be 


$$
\frac{W_{j}}{W_{i}}=\frac{\left\langle\frac{\Psi_{i}\left(\mathbf{r}^{N}\right)}{\pi\left(\mathbf{r}^{N}\right)}\right\rangle_{\pi\left(\mathbf{r}^{N}\right)}}{\left\langle\frac{\Psi_{j}\left(\mathbf{r}^{N}\right)}{\pi\left(\mathbf{r}^{N}\right)}\right\rangle_{\pi\left(\mathbf{r}^{N}\right)}} .
$$

In a practical sense, the averages in equation (7) are valid only if the sampling distribution $\pi\left(\mathbf{r}^{N}\right)$, used to collect the averages, visits states relevant to each of the Hamiltonians with sufficient frequency. This obstacle is overcome by using an iterative process. An initial guess is made for the sampling weights and a short simulation is completed, after which the weights of those Hamiltonians that were adequately sampled are updated. This process continues until a set of sampling weights are determined that sufficiently explore all relevant configurations.

Each Monte Carlo step consists of a randomly selected particle creation, annihilation, or displacement. The method of Metropolis et al. ${ }^{24}$ is used to propagate the Markov chain. Configuration $\mathbf{r}_{i}^{N}$ is advanced to configuration $\mathbf{r}_{i+1}^{N \pm 0,1}$ with probability

$$
P_{i \rightarrow i+1}=\min \left(1, \frac{\pi\left(\mathbf{r}_{i+1}^{N \pm 0,1}\right)}{\pi\left(\mathbf{r}_{i}^{N}\right)}\right)
$$

which ensures that configurations are generated according to the sampling distribution $\pi\left(\mathbf{r}^{N}\right)$.

When using histogram-reweighting techniques, during the course of a simulation, one needs to collect a histogram of the frequency of occurrence, 
$f(N, E)$, of $N$ particles with total configurational energy $E$. Note that the incorporation of scaling does not make every configuration equally relevant for all Hamiltonians, instead the fractional importance of each Hamiltonian for a given configuration is $\frac{W_{n} \Psi_{n}\left(\mathbf{r}^{N}\right)}{\pi\left(\mathbf{r}^{N}\right)}$. Thus, during a simulation, separate histograms are collected for each Hamiltonian with each bin calculated as follows

$$
f_{n}\left(N_{i}, E_{n, i}\right)=\sum_{\substack{M C \text { Steps } \\ N=N_{i}, E_{n}=E_{n, i}}}\left(\frac{W_{n} \Psi_{n}\left(\mathbf{r}^{N}\right)}{\pi\left(\mathbf{r}^{N}\right)}\right)_{\pi\left(\mathbf{r}^{N}\right)}
$$

The information contained in these histograms is then equivalent to one collected using conventional grand canonical Monte Carlo.

\section{Configurational-Bias Sampling}

For some systems, the creation and annihilation of particles can severely restrict the applicability of a grand canonical ensemble simulation. The insertion or deletion of a particle may cause too severe of an energy change to allow the transfer to occur. The efficiency of particle exchanges is improved when configurational-bias techniques ${ }^{25.28}$ are used. The method has been described in detail elsewhere ${ }^{29}$. Here we will simply expand the method to incorporate Hamiltonian scaling.

In general, a molecule is inserted in a series of $M$ steps. For the Buckingham exponential-6 potential only a single step is possible, so $M=1$. At the 
$l^{\text {th }}$ step $k_{l}$ trial positions or growth directions, denoted $\{\mathbf{b}\}_{k_{l}}=\mathbf{b}_{1}, \mathbf{b}_{2}, \ldots, \mathbf{b}_{k_{l}}$, are attempted and the quantity

$$
y_{n, l}=\sum_{j=1}^{k_{l}} \exp \left[-\beta_{n} e_{n, l}\left(\mathbf{b}_{j}\right)\right]
$$

is calculated for each Hamiltonian, where $e_{n, l}$ is the energy change during the $l^{\text {th }}$ step for Hamiltonian $n$. Out of the $k_{l}$ trial positions, one is selected with probability

$$
P_{l}\left(\mathbf{b}_{i}\right)=\frac{\sum_{n} W_{n} \exp \left[-\beta_{n} e_{n, l}\left(\mathbf{b}_{i}\right)\right]}{\sum_{n} W_{n} y_{n, l}} .
$$

When the entire molecule has been inserted the Rosenbluth factor of the molecule, $Y_{n}$, is calculated for each Hamiltonian

$$
Y_{n}=\prod_{l=1}^{M} y_{n, l}
$$

The new sampling distribution for step $i+1$ is then calculated from

$$
\pi\left(\mathbf{r}_{i+1}^{N+1}\right)=\sum_{n} W_{n} \Psi\left(\mathbf{r}_{i}^{N}\right) \frac{\exp \left(\beta_{n} \mu_{n}\right) V Y_{n}}{\Lambda_{n}^{3}(N+1)}
$$

and equation (8) is used to determine acceptance.

A particle is removed in the reverse direction from which it was grown. The Rosenbluth factor is calculated the same way as above with one exception. The current configuration is used as one of the $k_{l}$ positions or growth directions and thus only $k_{l}-1$ trials need to be generated at each step. The new sampling distribution is then found from, 


$$
\pi\left(\mathbf{r}_{i+1}^{N-1}\right)=\sum_{n} W_{n} \Psi_{n}\left(\mathbf{r}_{i}^{N}\right) \frac{\Lambda_{n}^{3} N}{\exp \left(\beta_{n} \mu_{n}\right) V Y_{n}}
$$

and again equation (8) is used to determine acceptance. The inclusion of intramolecular degrees of freedom is a straightforward extension of the material above combined with that of reference 29.

\section{Histogram-Reweighting}

Histogram-reweighting methods are used to provide information about a system at different chemical potentials and temperatures than the ones at which a simulation was performed ${ }^{8}$. Here, we review the techniques relevant to the current study. A two dimensional histogram, $f_{1}(N, E)$, is collected for a given chemical potential, $\mu_{1}$, and temperature, $T_{1}$, during a simulation for which

$$
f_{1}(N, E) \cdot C=\Omega(N, V, E) \exp \left(\beta_{1} \mu_{1} N-\beta_{1} E\right)
$$

where $C$ is a simulation specific constant. We note that it is irrelevant if the histogram is collected using conventional or Hamiltonian scaling grand canonical Monte Carlo. For a new simulation at a different chemical potential, $\mu_{2}$, and temperature, $T_{2}$, one would expect a new distribution, $f_{2}(N, E)$, with

$$
\frac{f_{2}(N, E)}{f_{1}(N, E)} \propto \exp \left[\left(\beta_{2} \mu_{2}-\beta_{1} \mu_{1}\right) N-\left(\beta_{2}-\beta_{1}\right) E\right] .
$$

The practical range of validity of equation (16) can be expanded by performing additional runs at different state points with overlapping particle number and energy distributions. Once the histograms are collected, they need to be combined into one unified distribution. Ferrenberg and Swedsen have 
developed a technique ${ }^{30}$ which ensures a minimum deviation between the predicted and observed histograms. The distribution $P_{\mu, \beta}(N, E)$ for a given number of particles and energy that results from combining $R$ runs is

$$
P_{\mu, \beta}(N, E)=\frac{\sum_{n=1}^{R} f_{n}(N, E) \exp [\beta \mu N-\beta E]}{\sum_{m=1}^{R} K_{m} \exp \left[\beta_{m} \mu_{m} N-\beta_{m} E-C_{m}\right]},
$$

where $K_{m}$ is the total number of observations for run $m$. The constants $C_{n}$ are obtained using an iterative relationship

$$
\exp \left[C_{n}\right]=\sum_{N} \sum_{E} P_{\mu_{n}, \beta_{n}}(N, E)
$$

Equation (17) is valid when all runs have the same statistical efficiency. The distribution $P_{\mu, \beta}(N, E)$ is equivalent to the distribution $f(N, E)$ determined from a simulation at chemical potential, $\mu$, and temperature, $T$.

Let us now turn our attention to the grand partition function. With knowledge of the grand partition function over a range of chemical potentials and temperatures, one can calculate the thermophysical properties of the system. The grand partition function is defined as

$$
\Xi(\mu, V, T)=\sum_{N} \sum_{E} \Omega(N, V, E) \exp [\beta \mu N-\beta E],
$$

as well as through the bridge equation

$$
\beta P V=\ln \Xi .
$$

By comparing equations (15) and (19) one realizes that the grand partition function is known to within a simulation-specific constant. To obtain the 
constant the system needs to be related to a known reference state. The ideal gas state is a convenient choice. Introducing the ideal gas equation of state $(\beta P=\rho)$ into the above equations leads to

$$
\langle N\rangle=\ln C+\sum_{N} \sum_{E} f(N, E) .
$$

The distribution $f(N, E)$ can be reweighted for a series of chemical potentials and temperatures that exhibit ideal gas behavior. The data collected can then be fitted to equation (21) to yield the constant $C$. With the grand partition function the equation of state for the fluid can directly be determined.

At subcritical temperatures phase coexistence in the grand canonical ensemble can be determined by finding a chemical potential that gives equal pressures in both phases. This is done by arbitrarily assigning a particle number which separates the liquid and vapor phases and finding a chemical potential which gives equal areas under the liquid and vapor $f(N, E)$ distributions. At near critical temperatures the liquid and vapor peaks no longer separate enough to allow the determination of coexistence with sufficient accuracy.

\section{Mixed-Field Finite-Size Scaling}

The mixed-field finite size scaling concepts of Wilding et al. ${ }^{10,11}$ were used to obtain estimates of the critical parameters. The theory accounts for liquidvapor asymmetry by defining an order parameter, $M$, as a combination of the density and energy fields

$$
M=N-s E,
$$


where $s$ is a system-specific field mixing parameter that controls the strength of coupling between energy and density fluctuations near the critical point. At the critical point the distribution, $P_{L}(x)$, with $x=a(L, r) \cdot\left(M-M_{c}\right)$, is assumed to be universal. The non-universal parameter $a(L, r)$ is chosen to result in a unit variance for the distribution $P_{L}(x)$. Simulations are performed at near critical point conditions. The distributions are then reweighted to find the chemical potential and temperature which yield a $P_{L}(x)$ which best matches the three dimensional Ising distribution.

\section{Simulation Details}

Simulations were performed to obtain the phase behavior of twelve Hamiltonians resulting from variations of parameters $\alpha$ and $C$ in equation (1), with $\alpha=\{12,14,16,18\}$ and $C=\{0.9,1.0,1.1\}$. The value of $r_{m}$ was set to unity for each of the Hamiltonians, and thus $\sigma$ took on different values for each Hamiltonian. The simulation cell volume was set to $V=125 \cdot r_{m}^{3}$ for all runs. Long-range corrections were applied using the Theodorou-Suter approach ${ }^{35}$ Eight sets of runs were completed that covered a reduced temperature $\left(T_{r}^{*}=T / T_{c}\right)$ range of $T_{r}^{*}=0.65$ to $T_{r}^{*}=1.0$. Over the course of the eight runs the density fluctuated between $\rho^{*}=0.0$ to $\rho^{*}=0.86$. Each set was divided into five blocks so that statistical uncertainties could be evaluated. A mixture of $40 \%$ particle displacements and $60 \%$ creation/annihilation steps was used. The 
number of trial positions for a creation/annihilation step varied between 1 for gas-like conditions, to 50 for low temperature, high density liquid conditions.

The study started with a run at near critical conditions. The $\alpha=14, C=1.0$ Hamiltonian was used as the base Hamiltonian. Preliminary runs were completed to establish the approximate location of the critical point for the base Hamiltonian. A series of steps were then completed to approximate the critical chemical potential and temperature for each of the other Hamiltonians. For the first step the weight of the base Hamiltonian was set to unity and the others set to zero. All the Hamiltonians were assigned the same chemical potential and temperature as the base Hamiltonian. A short simulation was completed, after which the $f(N)$ distribution of the eleven non-base Hamiltonians were reweighted using equation (16) to find a chemical potential and temperature for which the $f(N)$ distribution best matched that of the base Hamiltonian. The second step matched the first, with the exception that the chemical potentials and temperatures assigned were the approximate values found from step one. At the end of step two the distributions were again fit to the base Hamiltonian. In addition, the weights for all the Hamiltonians were updated using equation (7). The iterative process continued, with the chemical potentials, temperatures, and weights updated at the end of each step. The steps were progressively lengthened until the chemical potentials, temperatures, and weights became stable. The process was completed with eight iterative steps and $15 \times 10^{6}$ Monte Carlo steps. 
The near critical distributions were then used to determine the critical parameters and a limited region of the coexistence curve. It was found that the determination of phase coexistence became inaccurate below a reduced temperature $T_{r}^{*}=0.88$. A second and third set of simulations were then performed at a reduced temperature, $T_{r}^{*}=0.88$, and activities, $\zeta=\frac{\exp (\beta \mu)}{\Lambda^{3}}$, of $\ln \zeta=\ln \zeta_{c o e x} \pm 0.12$, where $\zeta_{c o e x}$ was the activity that gave phase coexistence. This corresponds to a liquid and vapor region just outside the phase envelope. Exploratory simulations were done to determine the weights. Unlike the nearcritical simulations, the activities and temperatures for each of the Hamiltonians were already set, so only the weights needed to be determined. The weights were obtained iteratively using equation (7). The new distributions were then combined with the critical distributions to further determine the coexistence curve. This process was repeated for a reduced temperature of $T_{r}^{*}=0.76$ and $T_{r}^{*}=0.65$. One further distribution was collected at a reduced temperature of $T_{r}^{*}=1.0$ and activity of $\zeta=\zeta_{c}+2.0$, where $\zeta_{c}$ was the critical activity, which produced a vapor peak with a maximum at one particle. This was done to ensure the ideal gas region was adequately sampled, and thus the constant of equation (21) accurately determined.

A typical run of $15 \times 10^{6}$ configurations near the critical point, with twelve Hamiltonians, took $6.7 \mathrm{CPU}$ hours on a $500 \mathrm{MHz}$ DEC Alpha processor. A similar run with only a single Hamiltonian took 1.3 CPU hours. Thus, the use of 
Hamiltonian scaling resulted in computational time savings of a factor of $1.3 \times 12 / 6.7=2.3$. A total of $75 \times 10^{6}$ configurations near the critical point were sampled.

\section{Results and Discussion}

\section{Critical Parameters}

The critical parameters of the twelve Hamiltonians simulated in this study are listed in Table 1. Non-dimensional variables are defined in the usual fashion: $T^{*}=k_{B} T / \varepsilon, \rho^{*}=N \sigma^{3} / V, P^{*}=P \sigma^{3} / \varepsilon$. A mixed-field finite-size scaling analysis was used to find the critical temperatures and densities. The critical pressures were found by extrapolating the vapor pressure curve to the critical temperature. The vapor pressures were fitted to the semi-empirical equation

$$
\log P^{s a t}=a_{0}+\frac{a_{1}}{T}+a_{2} T+a_{3} T^{2}
$$

from a reduced temperature of $T_{r}^{*}=0.80$ to the near critical region. Equation (23), truncated after the first two terms, is analogous to the Clausius-Clapeyron equation. The third and fourth terms on the right hand side of equation (23) were added as empirical correction factors. The critical pressures were found by evaluating equation (23) at a reduced temperature of one. Using this method, the critical temperatures were found to within $0.3 \%$ and the critical densities and critical pressures were found to within $1 \%$. 
Dodd and Sandler reported a critical temperature for the $C=1.0$ and $\alpha=14$ case of, $T_{c}^{*}=1.2696$, a few percent higher than our result. This comparison is reasonable, considering their prediction was based on a classical equation of state. The agreement in the critical pressure is not as good. The critical pressure reported by Dodd and Sandler, $P_{c}^{*}=0.1431$, is $25 \%$ larger than our prediction.

\section{Phase Behavior}

The phase diagrams for $C=1.0$ and $\alpha=\{12,14,16,18\}$ are shown in Figure 1. Near the critical point the coexistence densities were determined by fitting to the scaling relationships

$$
\frac{1}{2}\left(\rho_{L}+\rho_{V}\right)-\rho_{c}=A \tau^{\mu}
$$

and

$$
\rho_{L}-\rho_{V}=\tau^{\beta}\left(B_{0}+B_{1} \tau^{\theta}\right)
$$

with

$$
\tau=\frac{T_{c}-T}{T_{c}}
$$

The scaling exponents corresponding to the Ising universality class, $\beta=0.326$, $\mu=0.90$, and $\theta=0.54$, were employed ${ }^{31}$. The constants $A, B_{0}$, and $B_{1}$ were identified as the parameters that yielded the best fit curves. The reduced distance from the critical point over which coexistence data were fitted was $\tau \approx 0.85$. Over the temperature range sampled, the average uncertainty in the saturated liquid densities, saturated vapor densities, and vapor pressures was $0.34 \%, 0.50 \%$ and 
$0.37 \%$ respectively. Additional diagrams for different combinations of $C$ and $\alpha$ along with data sets for the twelve Hamiltonians can be found on the World Wide $\mathrm{Web}^{32}$.

The differences between the twelve Hamiltonians becomes evident when comparing the reduced thermodynamic properties. The reduced saturated liquid densities show only small differences. When keeping $C$ constant and varying $\alpha$, the saturated liquid densities become slightly larger with an increasing value of $\alpha$ for a given reduced temperature. When keeping $\alpha$ constant and varying $C$ no trends were observed. For the reduced saturated vapor densities and reduced vapor pressures the differences are larger. When keeping $C$ constant and varying $\alpha$, the vapor densities and vapor pressures decrease with an increasing value of $\alpha$ for a given reduced temperature. This corresponds to an increasing acentric factor ${ }^{33}$ with an increasing value of $\alpha$. This trend is shown in Figure 2 for the $C=1.0$ and $\alpha=\{12,14,16,18\}$ case. Again, no trends were observed when keeping $\alpha$ constant and varying $C$.

\section{Fit to Methane}

The final objective was to see how well the Buckingham exponential-6 potential could describe the phase behavior of a real fluid. We chose methane because it is a simple, nearly spherical non-polar substance. Clearly, a single-site Buckingham exponential-6 potential model is an imperfect "united-atom" representation of real methane. As noted above, there was little difference in the 
reduced phase behavior for varying values of $C$ with $\alpha$ held constant. Therefore, we decided to fix the value of $C$ at 1.0 and search for a value of $\alpha$ which best describes methane. To find the appropriate value of $\alpha$ we compared the reduced coexistence curve of methane to the reduced coexistence curves for $C=1.0$ and $\alpha=$ $\{12,14,16,18\}$. The experimental critical parameters of methane ${ }^{34}$ are $T_{c}=190.56$ $\mathrm{K}, \rho_{c}=162.66 \mathrm{~kg} / \mathrm{m}^{3}, P_{c}=45.992$ bar. The critical temperature was used to reduce temperatures. The critical density used to reduce the densities was varied until the reduced saturated liquid densities of methane matched those of the exponential-6 potentials. There was very little difference in the reduced saturated liquid densities for the different values of $\alpha$. The reduced saturated vapor densities were then compared to determine the best value for $\alpha$. It was determined that the most appropriate value of $\alpha$ was 15 , which lead to an $\varepsilon / k_{B}$ value of $160.3 \mathrm{~K}$ and an $r_{m}$ value of $4.188 \AA$ (which gives $\sigma=3.741 \AA$ ).

A similar technique was used to find parameters for the Lennard-Jones potential. The equation of state of Johnson et al. ${ }^{4}$, which was fitted to a large number of simulation results, was used to calculate phase coexistence data for the Lennard-Jones fluid. The equation is classical and produces mean field type behavior in the critical region instead of the correct Ising behavior. We found that a better representation of the coexistence properties of methane can be obtained by fitting the critical temperature, critical density and near critical phase behavior to the subcritical data of Johnson et al. using equations (24-26). A critical temperature of $T_{c}^{*}=1.2963$ and a critical density of $\rho_{c}^{*}=0.3167$ were 
found. An $\varepsilon / k_{B}$ value of $147.0 \mathrm{~K}$ was then selected in order to reproduce the experimental critical temperature of methane. The value of $\sigma$ was varied until the saturated liquid densities predicted by the Lennard-Jones potential matched those of methane. This resulted in a value for $\sigma=3.723 \AA$.

The resulting coexistence curve predicted by the Buckingham exponential6 and Lennard-Jones potentials are compared to experimental data ${ }^{34}$ in Figure 3. Both potentials do a good job describing the overall coexistence curve of methane. Figure 4 shows the vapor pressures predicted by the Buckingham exponential- 6 and Lennard-Jones potentials compared to experimental data ${ }^{34}$. In this representation, the relative effectiveness of the two potentials is evident. The Buckingham exponential-6 potential does a much better job describing the vapor pressures of methane than the Lennard-Jones potential. This point is further emphasized in Figure 5. Here, the percent deviation of the Buckingham exponential-6 potential and Lennard-Jones potential from experimental values are plotted as a function of temperature for the saturated liquid density, saturated vapor density, and vapor pressure. Over the temperature range selected the Buckingham exponential- 6 potential does not deviate by more than a few percent for any of the properties while the saturated vapor densities and vapor pressures predicted by the Lennard-Jones potential deviate by as much as fifteen percent at low temperatures. It is clear that the additional parameter of the Buckingham exponential-6 potential is needed in order to describe accurately a real fluid as simple as methane. 


\section{Conclusions}

Hamiltonian scaling grand canonical Monte Carlo has been introduced as a new technique for determining the critical parameters and phase behavior of many Hamiltonians (potential models) from a single set of simulations. The advantage this method has over current scaling techniques is that all of the Hamiltonians sample approximately the same densities during the simulation. This property translates into significant computer and researcher time savings. The method produces separate histograms for each of the Hamiltonians. Histogram reweighting and mixed-field finite-size scaling techniques are then applied to each histogram individually to determine the phase behavior and critical parameters for a given Hamiltonian.

The critical parameters for the modified Buckingham exponential-6 potential have been calculated to within $0.3 \%$ for the critical temperature and $1 \%$ for the critical density and critical pressure. The coexistence curves at subcritical temperatures were determined with average uncertainties of $0.34 \%$ for the liquid density, $0.50 \%$ for the vapor density, and $0.37 \%$ for the vapor pressure. This level of accuracy is not possible using the Gibbs ensemble method. It was determined that the $C$ parameter changes the reduced thermodynamic behavior by a relatively small amount. Conversely, the parameter $\alpha$ has a significant effect on the reduced thermodynamic behavior. It was shown that the greater flexibility of the Buckingham potential vastly increased the accuracy to which one could predict the phase coexistence curve and vapor pressure of a simple 
real fluid such as methane from a united-atom model. The accuracy of representation is significantly higher in comparison to the Lennard-Jones potential, at the cost of introducing one extra parameter in the potential model.

\section{Acknowledgments}

Financial support for this work has been provided by the National Science Foundation, under grant CTS-9509158. Computational resources were partly provided by the Cornell Theory Center. 


\section{References}

${ }^{1}$ W. W. Wood and F. R. Parker, J. Chem. Phys. 27, 720 (1957).

${ }^{2}$ L. Verlet, Phys. Rev. 159, 98 (1967).

${ }^{3}$ J. J. Nicolas, K. E. Gubbins, W. B. Street, and D. J. Tildesley, Mol. Phys. 37, 1429 (1979).

${ }^{4}$ J. K. Johnson, J. A. Zollweg, and K. E. Gubbins, Mol. Phys. 78, 591 (1993).

${ }^{5}$ A. Z. Panagiotopoulos, Mol. Phys. 61, 813 (1987).

${ }^{6}$ A. Z. Panagiotopoulos, N. Quirke, M. Stapleton, and D. J. Tildesley, Mol. Phys. 63, 527 (1988).

${ }^{7}$ B. Smit, P. de Smedt, and D. Frenkel, Mol. Phys. 68, 931 (1989).

${ }^{8}$ A. M. Ferrenberg and R. H. Swendsen, Phys. Rev. Lett. 61, 2635 (1988).

${ }^{9}$ A. Z. Panagiotopoulos and V. Wong, Macromolecules, 31, 912 (1998).

${ }^{10}$ N. B. Wilding and A. D. Bruce, J. Phys-Condens. Mat. 4, 3087 (1992).

${ }^{11}$ N. B. Wilding, Phys. Rev. E 52, 602 (1995).

${ }^{12}$ B. Smit, S. Karaborni, and J. I. Siepmann, J. Chem. Phys. 102, 2126 (1995).

${ }^{13}$ M. E. van Leeuwen and B. Smit, J. Phys. Chem. 99, 1831 (1995).

${ }^{14}$ G. C. Boulougouris, I. G. Economou, and D. N. Theodorou, J. Phys. Chem. B, 1021029 (1998).

${ }^{15}$ G. M. Torrie and J. P. Valleau, J. Comput. Phys. 23, 187 (1977).

${ }^{16}$ G. M. Torrie and J. P. Valleau, J. Chem. Phys. 66, 1402 (1977). 
${ }^{17}$ J. P. Valleau, J. Comput. Phys. 96, 193 (1991).

${ }^{18}$ J. P. Valleau, J. Chem. Phys. 99, 4718 (1993).

${ }^{19}$ J. P. Valleau, "Thermodynamic-scaling Methods in Monte Carlo and their Application to Phase Equilibria," Adv. Chem. Phys., in press (1998).

${ }^{20}$ K. Kiyohara, T. Spyriouni, K. E. Gubbins, and A. Z. Panagiotopoulos, Mol. Phys. 89, 965 (1996).

${ }^{21}$ R. A. Buckingham, Proc. Roy. Soc. 168A, 264 (1938).

${ }^{22}$ L. R. Dodd and S. I. Sandler, Mol. Simulat. 2, 15 (1989).

${ }^{23}$ L. R. Dodd and S. I. Sandler, Fluid Phase Equilibr. 63, 279 (1991).

${ }^{24}$ N. Metropolis, A. W. Rosenbluth. M. N. Rosenbluth, A. H. Teller, and E. Teller, J. Chem. Phys. 21, 1087 (1953).

${ }^{25}$ J. I. Siepmann, Mol. Phys. 70, 1145 (1990).

${ }^{26}$ J. I. Siepmann and D. Frenkel, Mol. Phys. 75, 59 (1992).

${ }^{27}$ D. Frenkel, G. C. A. M. Mooij, and B. Smit, J. Phys-Condens. Mat. 4, 3053 (1992).

${ }^{28}$ J. J. de Pablo, M. Laso, and U. W. Suter, J. Chem. Phys. 96, 2395 (1992).

${ }^{29}$ D. Frenkel and B. Smit, Understanding Molecular Simulation (Academic Press, London, 1996).

${ }^{30}$ A. M. Ferrenberg and R. H. Swendsen, Phys. Rev. Lett. 63, 1195 (1989).

${ }^{35}$ D.N. Theodorou and U.W. Suter, J. Chem. Phys. 82, 955 (1985).

${ }^{31}$ B. G. Nickel and J. R. Rehr, J. Stat. Phys. 61, 1 (1990). 
${ }^{32}$ http://thera.umd.edu/jerring/buck .

${ }^{33}$ P.L. Chueh and J.M. Prausnitz, Ind. Eng. Chem. Fundam., 6, 492 (1967).

${ }^{34}$ NIST Chemistry WebBook, http:/ / www.nist.gov/chemistry. 


\section{Tables}

Table 1 The critical parameters of the modified Buckingham exponential-6 potential. Simulation uncertainties are given in parentheses, in units of the last decimal figure listed.

\begin{tabular}{|c|c|c|c|}
\hline \multicolumn{4}{|c|}{ Critical Temperature, $T_{c}^{*}$} \\
\hline$\alpha \backslash C$ & 0.9 & 1.0 & 1.1 \\
\hline 12 & $1.1109(15)$ & $1.4039(29)$ & $1.7588(47)$ \\
\hline 14 & $1.0222(10)$ & $1.2430(11)$ & $1.4901(32)$ \\
\hline 16 & 0.9629 (16) & $1.1467(13)$ & $1.3459(20)$ \\
\hline 18 & $0.9195(10)$ & $1.0811(11)$ & $1.2530(22)$ \\
\hline \multicolumn{4}{|c|}{ Critical Density, $\rho_{c}^{*}$} \\
\hline$\alpha \backslash C$ & 0.9 & 1.0 & 1.1 \\
\hline 12 & $0.3167(20)$ & $0.3163(16)$ & $0.3128(19)$ \\
\hline 14 & $0.3224(22)$ & $0.3200(29)$ & $0.3184(15)$ \\
\hline 16 & $0.3286(22)$ & $0.3268(20)$ & $0.3245(24)$ \\
\hline 18 & 0.3315 (19) & $0.3328(20)$ & $0.3306(13)$ \\
\hline \multicolumn{4}{|c|}{ Critical Pressure, $P_{c}^{*}$} \\
\hline$\alpha \backslash C$ & 0.9 & 1.0 & 1.1 \\
\hline 12 & $0.0998(7)$ & 0.1295 (13) & 0.1709 (19) \\
\hline 14 & $0.0923(6)$ & $0.1134(4)$ & $0.1377(15)$ \\
\hline 16 & $0.0885(10)$ & $0.1052(7)$ & $0.1239(10)$ \\
\hline 18 & $0.0859(7)$ & $0.1007(7)$ & 0.1165 (12) \\
\hline
\end{tabular}




\section{Figure Captions}

Figure 1 Phase diagrams of the modified Buckingham exponential-6 potential with $C=1.0$. The curves from top to bottom are for $\alpha=12,14,16$, and 18 .

Figure 2 Vapor pressures of the modified Buckingham exponential-6 potential with $C=1.0$. The lines from top to bottom are for $\alpha=12,14,16$, and 18 .

Figure 3 Phase diagram of methane. The open circles represent the Buckingham exponential-6 potential $(\alpha=15)$ and the squares represent the Lennard-Jones potential. The other potential parameters are given in the text.

Figure 4 Vapor pressure of methane. The open circles represent the Buckingham exponential-6 potential $(\alpha=15)$ and the squares represent the Lennard-Jones potential.

Figure 5 Percent deviation from experiment for the saturated liquid densities, saturated vapor densities, and vapor pressures for methane. The open symbols represent the Buckingham exponential-6 potential and the filled symbols represent the Lennard-Jones potential. The stars are for the vapor pressures, the triangles are for the saturated vapor densities, and the circles are for the saturated liquid densities. 